\title{
THE NORTHERN LIGHTS AS AN INTRODUCTION TO THE UNIVERSE
}

\author{
J.E. Solheim \\ Institute of Mathematical and Physical Sciences, University of Troms $\varnothing$, Norway
}

\section{Introduction}

The University of Troms $\emptyset$ is located at $70^{\circ}$ northern latitude. It is planned for 3,000 students and was opened in 1972. Research interests in physics are centered on the physics related to the upper atmosphere. The region is coastal and mountainous, and is sparsely inhabited. People in the region are good observers, and those away from city lights watch the lively auroral displays that can be observed almost every clear, dark night. The different auroral forms - wavering structure, colors, and diffusing and changing appearance - all challenge the imagination. There are popular and folkloric descriptions as well as manifestations in art, including poetry, paintings, music, and drama.

\section{The Course}

An introductory course in astronomy and space-science physics has been developed - using the northern lights as the central theme - guiding us from our planet to the most distant parts of the universe.

The aurora borealis is the end effect of a solar wind blowing charged particles from the sun and interacting with the Earth's magnetic field. The magnetosphere acts like a large dynamo - producing the power to accelerate particles - just as in a cathode ray tube. The equivalent of the CRT screen is the ring around the magnetic poles where the magnetic lines open to space. The colors are due to emission lines from atomic oxygen or nitrogen at altitudes $90 \mathrm{~km}$ or higher. The same phenomenon can be predicted and observed on other planets that have a magnetic field and an atmosphere. The solar wind also interacts with comets and other bodies in the solar system. More energetic winds can be observed from stars of other types and stages in their development.

Finally, interacting binary stellar systems that contain a compact object a white dwarf, a neutron star, or a black hole - as an accreter, show what happens when gravitation assists in the process of pulling off matter and dominates in the accretion process itself. We observe the high-energy version of the aurora as ultraviolet radiation, X-rays, or gamma rays from interacting compact systems.

To understand the source, the sun, we have to study the sun's interior, core, magnetic properties, cycles, twist of magnetic field lines, and how energy is transported from the interior through the atmosphere and the corona to the interplanetary medium. Coronal holes and magnetic field lines help us find the way, and we can discuss the life of a charged particle from the sun to the ignition of the auroral 
display.

We compare the sun with other active stars. We explain stellar evolution to show how stars interact with their environment, and lead up to the supernovae as the most dramatic space polluter. We also look at the powerful emitters in the center of galaxies producing quasars and gigantic cosmic jets. At the end of the course, we ask whether similar processes on an extraordinary scale were present in the moment of creation of the universe, when strings and superstrings may have been the prime actors on the scene.

Back on Earth, we study how auroral flames in the sky are interpreted through folkloric and scientific history. We find legends and tales, poetry and music, drawings and paintings, and certainly influence in the great epics of the people in the auroral zones. Students of art are invited to make projects concentrating on these aspects. Studying the history of science and asking how scientists interpreted the aurora during different periods is a fascinating topic by itself. Aristotle, Kepler, Galileo, Halley, and other famous astronomers and physicists had explanations of the aurora that reflected the development of science in their particular time in a very illustrative way.

In the future, we may demonstrate how it is possible from space to investigate the particles blowing from the sun and to check how the turbulence - which can vary from a gentle and steady solar breeze to a hurricane - can create problems for future space habitats. We can also study the aurora itself as seen from above, and even detect in it the daytime (in the ultraviolet). It may also be possible to find out if energy from space deposited in our atmosphere, through the action of our magnetospheric dynamo, affects the lower part of the atmosphere and the weather and climate on Earth. At this stage in the course, we have an excursion to a nearby rocket launching facility to see how rockets can be used to explore the atmosphere up to the height of satellites.

When we finish this journey searching for the explanation of the aurora, and its relatives on other energy scales, we find that we have been through most of the universe and a fair part of the physics known today.

\section{Related Activities}

Based on our experiences from this course, we are in the process of providing study material for high schools, in which auroral physics may be introduced as a special theme in physics. For the general public, and for the visitors to the region (who mostly come in the summer to see the fascinating midnight sun), we are in the process of construction a planetarium. In it northern lights will be demonstrated and explained, and related to the universe as described above.

\section{Additional Reading}

Seymour, P.: "Cosmic Magnetism," (Bristol and Boston, Adam Hilger) 1986.

Solheim, J.E.: "The Connection between the Earth and the Universe made visible by the Northern Lights," GIREP Conference 1986, Cosmos - An Educational 
Challenge, European Space Agency SP-253, 103-111.

Video showing the real movements of the aurora. (27 minutes in color): (NTSC): Aurora color television project, Room 413, University of Alaska, Fairbanks, Alaska 99775-0800, U.S.A. (PAL): Troms $\varnothing$ Museum, N-9000 Troms $\varnothing$, Norway.

\title{
Discussion
}

J.L. Dunlap: I have wondered if the Northern Lights have been mentioned or explained in myth or legends of the northern cultures? (Is, perhaps, the description of Grendel in Beowulf an allusion to the phenomenon of the lights, and the death an attempt to end fear of the phenomenon among the people?)

J.E. Solheim: Among all people in the north we find myths and legends related to the northern lights. In most cases it is connected with fear, but some places (Scotland, Sweden, and some Indian tribes) also connected with dances performed by heavenly creatures. It is not easy to connect the story of Grendel in Beowulf with the northern lights - since Grendel is a sea monster.

\section{ASTRONOMY FOR BUSINESS STUDENTS: Space Industrialization and the Commercial Potential of Space Technology}

\author{
James R. Philips \\ Math/Science Division, Babson College, Babson Park, \\ Wellesley, Massachusetts 02157, U.S.A.
}

When teaching science to nonmajors lacking an interest in science, two major goals are to stimulate their interest and to provide these students with information and scientific skills useful in their lives and careers. Business students now comprise over 23 per cent of the undergraduates in America, and they generally view science, including astronomy, as not relevant to their lives and careers. I find the students entering my introductory astronomy course for business students expect a pictorial tour of the universe, and are unhappy when asked to calculate redshifts in the laboratory or to attend an extra class meeting for telescope observing. Astronomy is not what they have come to a business college to learn, but they have a laboratoryscience requirement to fill for their degree.

The challenge is to educate these future corporate leaders so that they understand how science influences business, and can deal with the problems resulting from the rapid pace of technological innovation in the marketplace. Nonmajors' textbooks in astronomy, as well as in other sciences, are written to appeal to a 